\title{
Ulcerative Colitis with and without Primary Sclerosing Cholangitis: Two Different Diseases?
}

\author{
Atsushi Tanaka $^{\mathrm{a}}$ Joachim C. Mertens ${ }^{\mathrm{b}}$ \\ ${ }^{a}$ Department of Medicine, Teikyo University School of Medicine, Tokyo, Japan; ${ }^{b}$ University Hospital Zurich, \\ Zurich, Switzerland
}

\section{Key Words}

Ulcerative colitis · Primary sclerosing cholangitis

\begin{abstract}
Background: Primary sclerosing cholangitis (PSC) is a chronic cholestatic liver disease of unknown origin and an important hepatobiliary complication of inflammatory bowel diseases (IBD), especially ulcerative colitis (UC). When further differentiated, about 3-8\% of UC patients suffer from PSC, whereas among Crohn's disease patients the reported prevalence of PSC is probably between 1 and 3.5\%. Although it was reported from Japan that the frequency of PSC in UC was only $34 \%$, the same registry data indicated that the prevalence was up to $57 \%$ among young patients with PSC even in Japan, which is comparable to the $60-80 \%$ in Europe and the US. Additionally, the clinical features of UC in patients with PSC are different from those in patients without PSC, for instance, rectal sparing and right-sided dominance. Summary: The strong link between atypical IBD and PSC suggests that
\end{abstract}

\section{A.T. and J.C.M. contributed equally to this work.}

\section{KARGER}

() 2016 S. Karger AG, Base

2296-9403/16/0011-0009\$39.50/0

E-Mail karger@karger.com

www.karger.com/iid the pathogenesis of PSC involves pathology of the gut, including abnormal gut microbiota and aberrant activation of mucosal lymphocytes. These seem to be different in UC PSC as compared to the pathology of typical UC. Key Messages: The key to solving the question 'Is there a difference between East and West?' are genetic studies, genome-wide association studies of PSC in particular, which have already been performed in the West and are strongly warranted in the East.

๑) 2016 S. Karger AG, Basel

\section{Background West}

In Europe, especially Northern Europe, about 70-80\% of patients with primary sclerosing cholangitis (PSC) are also diagnosed with inflammatory bowel disease (IBD). This is primarily ulcerative colitis (UC), less frequently Crohn's disease (CD) [1]. Conversely, abnormalities of the hepatobiliary systems are found in up to $30 \%$ of IBD patients [2]. 
Of the liver diseases specifically associated with IBD, PSC is the most common, affecting up to $5 \%$ of all IBD patients depending on the geographic region [3]. When further differentiated, about $3-8 \%$ of UC patients suffer from PSC $[4,5]$, while in CD patients the reported prevalence of PSC is probably between 1 and 3.5\% [6]. PSC has a clear male predominance with a ratio of $2: 1$.

Prior to the diagnosis of PSC in a patient with elevated serum markers of cholestasis (alkaline phosphatase and $\gamma$-glutamyltransferase), a possible secondary sclerosing cholangitis caused, for example, by infection, ischemia, IgG4-related cholangiopathy, or autoimmune hepatitis needs to be excluded. While PSC may present with symptoms like fever, night sweats, and pruritus, it is important to remember that IBD patients are frequently asymptomatic with regard to their liver disease, and physicians treating IBD patients need to actively investigate for possible associated hepatobiliary conditions [7]. The diagnostic gold standard for PSC remains magnetic resonance cholangiography (MRC), with typical 'string-of-pearls' imaging features of bile duct changes with multifocal strictures and segmental dilatations. MRC findings may be complemented by histology from liver biopsy if imaging is inconclusive. Histologically, PSC can be differentiated by several staging systems. The Ludwig staging system applies 4 stages to describe the histological features of PSC: cholangitis and portal inflammation (stage I), periportal inflammation or fibrosis (stage II), septal fibrosis and/or bridging necrosis (stage III), and biliary cirrhosis (stage IV). The applicability and prognostic value of the histological scoring was just recently confirmed by de Vries et al. [8]. Patients that present with clinical, biochemical, and histological findings indicating PSC but show a normal cholangiogram in MRC are classified as having small-duct PSC [9]. Small-duct PSC seems to have differing HLA associations, depending on whether it occurs in the context of IBD or not. While small-duct PSC in IBD is associated with HLA-B*08 and thus seems to be an early or mild form of large-duct PSC, small-duct PSC without IBD displays a different association with HLA-DRB1*13:01 and may represent a different entity of inflammatory cholangitis. In general, small-duct PSC may have a better prognosis than large-duct PSC [10].

Currently there is no therapy for PSC available that has been shown to alter the course of the disease. Guidelines in Europe and the US differ regarding the use of ursodeoxycholic acid (UDCA), which has been shown to improve laboratory values and histology in PSC pa- tients when given at doses not higher than $15 \mathrm{mg} / \mathrm{kg} /$ day $[11,12]$. Unfortunately, UDCA has no proven benefit for transplant-free and overall survival. 24-norUrsodeoxycholic acid is a novel, modified derivative of UDCA currently in clinical trial after promising results in a PSC animal model [13].

Focusing on the characteristics of UC associated with PSC, it was noted that the course of the IBD is more often benign, with mild-to-quiescent clinical symptoms and possibly even normal endoscopic appearance that might result in underdiagnosis of UC in PSC patients [14]. Yet, pancolitis and rectal sparing are described more frequently, that is, in about 65 and 30\%, respectively, of UC PSC patients, compared to 50 and $10 \%$ of UC patients without PSC. Backwash ileitis may occur slightly more often in UC PSC (17 vs. $12 \%)$, but the data are controversial $[4,15,16]$. Possibly due to the milder and sometimes subclinical course of the IBD despite pancolitis, the risk of colorectal cancer (CRC) seems to be substantially higher in UC PSC. Alternative theories implicate impaired hepatic excretion of bile acids with subsequent accumulation of potentially mutagenic secondary bile acids in the colon $[17,18]$. The fact that CRC in UC PSC develops more frequently in the right hemicolon with the highest concentration of secondary bile acids could support this proposed pathogenetic mechanism, as could the potential protective effect of UDCA $[16,19]$. CRC in UC PSC seems to develop earlier in the course of the disease, with a mean interval from IBD diagnosis to CRC of 12-20 years compared to 17-44 years in non-PSC IBD, but a possible lead time bias due to earlier screening in UC PSC is being debated.

The striking association of PSC with UC has sparked numerous investigations into the underlying pathogenetic mechanisms, giving rise to several theories that link the two diseases to each other. While both are considered an immune-mediated condition, PSC lacks features of classic autoimmune diseases, beginning with its male predominance and the lack of response to any immunosuppressive therapy. Moreover, no disease-specific autoantibody has been identified for PSC [20].

There is mounting evidence that genetic susceptibility plays an important role in the development of both diseases and may constitute a strong link between them. There is an up to 40-fold increased risk of PSC in 1stdegree relatives when compared to the general population, supporting the concept of genetic susceptibility. Moreover, strong associations with HLA genes have been described earlier. In a recent genome-wide asso- 
ciation study (GWAS), a total of 16 strong susceptibility loci for PSC were identified, 12 of which were found outside the HLA region. There was a genetic overlap with known IBD susceptibility loci in only half of the identified PSC risk loci, indicating a similar but distinct genetic architecture. Likewise, $50 \%$ of the PSC loci overlap with susceptibility loci for other autoimmune diseases. For the PSC-IBD-overlapping loci there was a greater similarity between PSC and UC than between PSC and $\mathrm{CD}$, indicating a greater genetic similarity between PSC and UC. This is consistent with the much more frequent clinical association [21]. Importantly, the newly identified genetic risk factors account for no more than $10 \%$ of the estimated overall PSC susceptibility, again raising the important question of environmental factors for the PSC pathogenesis.

In addition to the firmly established genetic association between PSC and IBD there are the long-standing hypotheses of 'leaky gut' and 'toxic bile', which may likewise contribute to the close relationship between PSC and UC. The chronically inflamed and disturbed intestinal barrier in the setting of UC may facilitate a 'leakage' of bacterial components into the portal circulation and subsequent portal inflammation. The hepatic overload with products from the intestinal microbiota may also cause 'aberrant lymphocyte homing' of gut-restricted T cells into the liver $[22,23]$. This immunological mechanism of liver injury may be complemented by an altered bile composition in PSC, leading to more toxic bile with a resulting local injury to bile duct epithelia.

\section{Key Messages West}

PSC and UC are strongly associated in Europe and the US. Recent GWAS show a substantial genetic overlap while confirming distinct disease entities. The clinical course of UC with PSC is often oligosymptomatic compared to UC without PSC. Nonetheless, patients with UC PSC exhibit an increased risk for CRC. There currently is no effective therapy to alter the outcome of PSC.

\section{Conclusions West}

PSC with UC likely represents a distinct disease variant that requires special clinical surveillance. Further research into the gut-liver axis is needed to better understand and potentially treat this disease complex.

Ulcerative Colitis with and without Primary Sclerosing Cholangitis

\section{Background East}

PSC is a chronic cholestatic liver disease of unknown origin, characterized by the progressive destruction of bile ducts caused by diffuse inflammation and fibrosis, which eventually leads to liver cirrhosis [24]. PSC is believed to be a rare disease in Asia compared to Europe and North America. In fact, population-based epidemiological data on PSC are lacking in Asia; since 1996 there have been several small case series from India [25], Singapore [26], Korea [27], and Iran [28], each of them enrolling 10-20 patients with PSC. In the study from India [25], 18 patients were presented, and their clinical profiles including age (mean age 39.0 years) and male predominance were comparable to those in the West. IBD were found in $50 \%$ of the patients. The outcomes were poor; 4 patients died during the 20.1-month follow-up period. The study from Singapore [26] had enrolled 10 cases with PSC (mean age 50.9 years), confirming the male predominance; however, the outcome was better, with only 1 death during 6.6 years of follow-up. The only epidemiological data available in Asia are data from our group presenting the prevalence of PSC in Japan, as reported in 2014 [29]. According to this work and other previously reported data from the West, incidence and prevalence rates of PSC are summarized in table 1. Except in the study conducted in 1994, the prevalence of PSC was around 5-16 per 100,000 inhabitants in the West, compared to less than 1.0 in Japan. Although the reason for this difference is unclear, genetic factors clearly play a crucial role.

In Japan, we performed questionnaire-based, nationwide surveys for PSC in 2012 and 2015. According to this study [29], several characteristic features of Japanese PSC patients, which had been scarcely demonstrated before by epidemiological studies from other countries, were demonstrated. First, there were 2 peaks in age distribution at diagnosis, that is, in the $3 \mathrm{rd}$ and 7 th decades of life (fig. 1). Most of the previous epidemiological studies from North America and Europe indicated a single age group for the highest risk of developing PSC [30-33], with the exception of one study in Canada, which suggested two age groups (18-35 and $>65$ years) as being at higher risk, similar to what has been found in Japanese studies [34]. Second, the prevalence of IBD among PSC patients appeared to be low compared to that in Europe and North America, as described below. The estimated median survival was 13.1 years, and 5-year survival of all patients was $74.5 \%$. Multivariate analysis demonstrated that age $(\mathrm{OR}=1.73$, $1.09-2.75, \mathrm{p}=0.0206)$ and total bilirubin $(\mathrm{OR}=2.32$, 


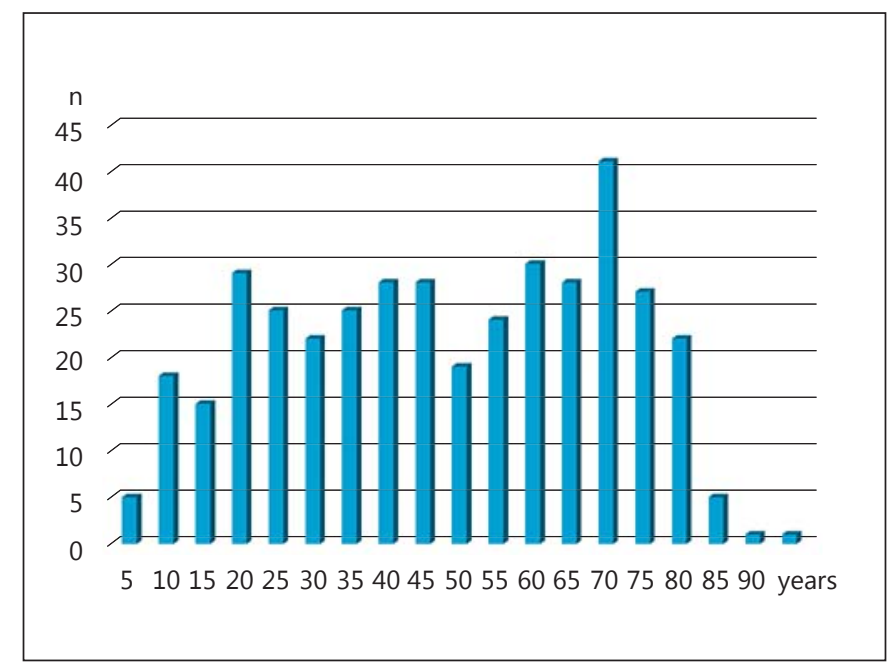

Fig. 1. Age distribution at presentation of patients with PSC in Japan.

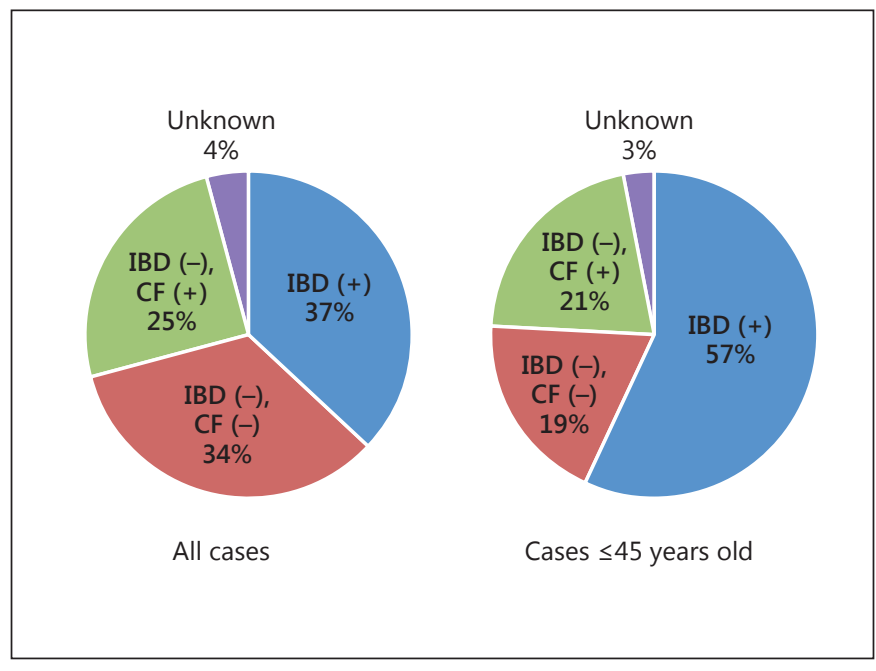

Fig. 2. Prevalence of IBD in patients with PSC in Japan. CF = Colonofiberscope.

Table 1. Incidence and prevalence of PSC

\begin{tabular}{|c|c|c|c|c|c|c|}
\hline Escorsell [36], 1994 & Spain & $1984-1988$ & Questionnaires & 43 & 0.07 & 0.22 \\
\hline Boberg [38], 1998 & Norway & $1986-1995$ & Prospective cohort & 17 & $1.3(0.8-2.1)$ & $8.5(2.8-14.2)$ \\
\hline Bambha [39], 2003 & USA & $1976-2000$ & Medical record linkage system & 22 & 0.9 & 13.6 \\
\hline Card [40], 2008 & UK & $1987-2002$ & General Practice Research Database & 149 & $0.41(0.34-0.48)$ & $3.85(3.04-4.80)$ \\
\hline Kaplan [43], 2007 & Canada & $2000-2005$ & Retrospective cohort & 49 & 0.92 & N/A \\
\hline Boonstra [44], 2013 & The Netherlands & $2000-2007$ & Retrospective cohort & 519 & 0.5 & 6.0 \\
\hline Tanaka [45], 2008 & Japan & 2007 & Questionnaires & 415 & N/A & $0.95(0.61-1.29)$ \\
\hline
\end{tabular}

N/A = Not available. ${ }^{a}$ Per 100,000 inhabitants.

1.47-3.66, $\mathrm{p}=0.0003$ ) were independent risk factors for the outcome of PSC in Japan [35].

PSC is an important hepatobiliary complication of UC. According to the reports from the West, PSC is observed as a comorbidity in $2.9-7.6 \%$ of patients with UC [7]. In the East, case series from Iran [28] and South Korea [27] demonstrated that the frequency of PSC among UC cases was $4.3 \%(19 / 447)$ and $1.1 \%(21 / 1,849)$, respectively, suggesting a comparable prevalence of PSC among patients with UC. Regarding patients with PSC, the prevalence of UC was striking: up to $80 \%$ of PSC patients have concomitant IBD. However, there appears to be a considerable geographical variation. In the study from Singapore [26],
IBD was found in $20 \%$ of patients with PSC, although only 10 cases were included. In our nationwide study in Japan, however, the prevalence of IBD as a comorbidity was low in Japanese PSC patients [29]. The presence of IBD was restricted to only $34 \%(68 / 197)$ of PSC patients. However, there are considerable differences in the prevalence of IBD between young and elderly patients. Their mean age was 48.1 years, and when comparing patients younger than 48.1 years with elderly patients, the prevalence of IBD was $12 \%$ in the elderly and $57 \%$ in the young PSC patients, the latter rate being comparable to the prevalence rates in $\mathrm{Eu}$ rope and the US (fig. 2). Taken together, the phenotype of PSC in the younger generation is similar to that in the 
West, while PSC in the elderly is a somewhat different disease and might not be 'primary' but 'secondary' sclerosing cholangitis.

It is also of note that, from Japanese experience, the clinical features of UC in patients with PSC are different from those in patients without PSC. It is known that several unique features were noted, such as rectal sparing and right-sided dominance, in UC patients with PSC. In addition, nonspecific or unclassified colitis is frequently found in patients with PSC. Interestingly, this atypical $\mathrm{UC}$ or IBD is also reported in a retrospective cohort study of IBD patients in Korea [27], as well as in North Ameri$\mathrm{ca}$ and European countries. Thus, the atypical presentation of IBD in PSC patients appears to be a general phenomenon worldwide. Furthermore, the strong link between atypical IBD and PSC suggests that the pathology of PSC clearly involves pathology of the gut, including abnormal gut microbiota and aberrant activation of mucosal lymphocytes, which seems to be different from the pathology of typical IBD or UC.

Obviously, the key to solving the question 'Is there a difference between East and West?' are genetic studies, GWAS of PSC in particular. While GWAS in patients with PSC were performed in the West and they indicated several susceptibility loci contributing to the development of PSC overlapping with those for IBD and other autoimmune diseases, no GWAS have been performed in the East. On another cholestatic liver disease, primary biliary cholangitis, GWAS have been performed both in the East and the West, demonstrating the involvement of similar pathways in the pathogenesis of primary biliary cholangitis, even though the susceptibility genes themselves were different, but there seemed to be no difference in genetic background between East and West. In Japan, GWAS using both young and elderly PSC patients are needed, and results should be analyzed and compared between younger PSC (with high complication rates of IBD) and elderly PSC patients (with low rates of IBD). Clinical experience suggests that the susceptibility loci in young PSC patients are similar to those observed in the West and overlapping with those in IBD or autoimmune diseases as well, while the susceptibility loci in the elderly are quite different or even undetectable.

\section{Key Messages East}

Japanese epidemiological data indicate that the prevalence of UC in patients with PSC appears to be low. However, in younger patients with PSC the prevalence of UC is $50-60 \%$, comparable to that in Europe and the US. It is reasonable to assume that in the younger generation, the phenotype of PSC is similar to that in the West. It is also of note that the clinical features of UC in patients with PSC are different from those in patients without PSC, for instance, rectal sparing and right-sided dominance and nonspecific or unclassified colitis.

\section{Conclusions East}

Genetic studies - GWAS for PSC in particular - are strongly warranted to solve the question whether there is a difference between PSC in the West and PSC in the East. Clinical experience suggests that the susceptibility loci in young PSC patients are similar to those observed in the West and overlapping with those in IBD or autoimmune diseases as well, while the susceptibility loci in the elderly are quite different or even undetectable.

\section{Disclosure Statement}

The authors have nothing to disclose.

\section{References}

1 Karlsen TH, Boberg KM: Update on primary sclerosing cholangitis. J Hepatol 2013;59: 571-582.

2 Mendes FD, et al: Abnormal hepatic biochemistries in patients with inflammatory bowel disease. Am J Gastroenterol 2007;102: 344-350.

- 3 Fausa O, Schrumpf E, Elgjo K: Relationship of inflammatory bowel disease and primary sclerosing cholangitis. Semin Liver Dis 1991; 11:31-39.

Ulcerative Colitis with and without Primary Sclerosing Cholangitis
4 Olsson R, et al: Prevalence of primary sclerosing cholangitis in patients with ulcerative colitis. Gastroenterology 1991;100:1319-1323.

5 Saich R, Chapman R: Primary sclerosing cholangitis, autoimmune hepatitis and overlap syndromes in inflammatory bowel disease. World J Gastroenterol 2008;14:331-337.

6 Rasmussen $\mathrm{HH}$, et al: Hepatobiliary dysfunction and primary sclerosing cholangitis in patients with Crohn's disease. Scand J Gastroenterol 1997;32:604-610.
7 Kummen M, Schrumpf E, Boberg KM: Liver abnormalities in bowel diseases. Best Pract Res Clin Gastroenterol 2013;27:531-542.

$\checkmark 8$ de Vries EM, et al: Applicability and prognostic value of histologic scoring systems in primary sclerosing cholangitis. J Hepatol 2015; 63:1212-1219.

$\checkmark$ European Association for the Study of the Liver: EASL Clinical Practice Guidelines: management of cholestatic liver diseases. J Hepatol 2009;51:237-267. 
10 Naess S, et al: Small duct primary sclerosing cholangitis without inflammatory bowel disease is genetically different from large duct disease. Liver Int 2014;34:1488-1495.

$\checkmark 11$ Beuers U, et al: Ursodeoxycholic acid for treatment of primary sclerosing cholangitis: a placebo-controlled trial. Hepatology 1992;16: 707-714.

$\checkmark 12$ Lindor KD: Ursodiol for primary sclerosing cholangitis. Mayo Primary Sclerosing Cholangitis-Ursodeoxycholic Acid Study Group. N Engl J Med 1997;336:691-695.

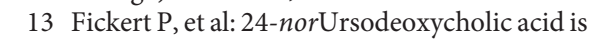
superior to ursodeoxycholic acid in the treatment of sclerosing cholangitis in $M d r 2$ (Abcb4) knockout mice. Gastroenterology 2006;130:465-481.

14 Jørgensen KK, et al: Inflammatory bowel disease in patients with primary sclerosing cholangitis: clinical characterization in liver transplanted and nontransplanted patients. Inflamm Bowel Dis 2012;18:536-545.

-15 Sinakos E, et al: Inflammatory bowel disease in primary sclerosing cholangitis: a robust yet changing relationship. Inflamm Bowel Dis 2013;19:1004-1009.

16 de Vries AB, et al: Distinctive inflammatory bowel disease phenotype in primary sclerosing cholangitis. World J Gastroenterol 2015; 21:1956-1971.

17 Torres J, et al: Review article: colorectal neoplasia in patients with primary sclerosing cholangitis and inflammatory bowel disease. Aliment Pharmacol Ther 2011;34:497-508.

18 Bernstein $\mathrm{H}$, et al: Bile acids as carcinogens in human gastrointestinal cancers. Mutat Res 2005;589:47-65.

19 de Valle MB, Björnsson E, Lindkvist B: Mortality and cancer risk related to primary sclerosing cholangitis in a Swedish populationbased cohort. Liver Int 2012;32:441-448.

-20 Krones E, et al: Evolving concepts in primary sclerosing cholangitis. Liver Int 2012;32:352369.

21 Liu JZ, et al: Dense genotyping of immunerelated disease regions identifies nine new risk loci for primary sclerosing cholangitis. Nat Genet 2013;45:670-675.
22 Adams DH, Eksteen B: Aberrant homing of mucosal $\mathrm{T}$ cells and extra-intestinal manifestations of inflammatory bowel disease. Nat Rev Immunol 2006;6:244-251.

23 Weismüller TJ, et al: The challenges in primary sclerosing cholangitis - aetiopathogenesis, autoimmunity, management and malignancy. J Hepatol 2008;48(suppl 1):S38-S57.

24 Hirschfield GM, et al: Primary sclerosing cholangitis. Lancet 2013;382:1587-1599.

25 Kochhar R, et al: Primary sclerosing cholangitis: an experience from India. J Gastroenterol Hepatol 1996;11:429-433.

26 Ang TL, et al: Clinical profile of primary sclerosing cholangitis in Singapore. J Gastroenterol Hepatol 2002;17:908-913.

27 Ye BD, et al: Clinical characteristics of ulcerative colitis associated with primary sclerosing cholangitis in Korea. Inflamm Bowel Dis 2011;17:1901-1906.

28 Khosravi Khorashad A, et al: Frequency and risk factors of primary sclerosing cholangitis among patients with inflammatory bowel disease in North-East of Iran. Gastroenterol Hepatol Bed Bench 2015;8:200-206.

29 Tanaka A, et al: Nationwide survey for primary sclerosing cholangitis and IgG4-related sclerosing cholangitis in Japan. J Hepatobiliary Pancreat Sci 2014;21:43-50.

30 Broome U, et al: Natural history and prognostic factors in 305 Swedish patients with primary sclerosing cholangitis. Gut 1996;38: 610-615.

-31 Farrant J, et al: Natural history and prognostic variables in primary sclerosing cholangitis. Ganstroenterology 1991;100:1710-1717.

-32 SchrumpfE, et al: Risk factors in primary sclerosing cholangitis. J Hepatol 1994;21:10611066.

33 Wiesner R, et al: Primary sclerosing cholangitis: natural history, prognostic factors and survival analysis. Hepatology 1989;10:430436.

34 Kaplan GG, et al: The burden of large and small duct primary sclerosing cholangitis in adults and children: a population-based analysis. Am J Gastroenterol 2007; 102:10421049.
35 Tanaka A, et al: Outcome and prognostic factors of 391 Japanese patients with primary sclerosing cholangitis. Liver Int 2008;28:983989.

36 Escorsell A, et al: Epidemiology of primary sclerosing cholangitis in Spain. Spanish Association for the Study of the Liver. J Hepatol 1994;21:787-791.

37 Berdal JE, Ebbesen J, Rydning A: Incidence and prevalence of autoimmune liver diseases (in Norwegian). Tidsskr Nor Laegeforen 1998;118:4517-4519.

38 Boberg KM, et al: Incidence and prevalence of primary biliary cirrhosis, primary sclerosing cholangitis, and autoimmune hepatitis in a Norwegian population. Scand J Gastroenterol 1998;33:99-103.

39 Bambha K, et al: Incidence, clinical spectrum, and outcomes of primary sclerosing cholangitis in a United States community. Gastroenterology 2003;125:1364-1369.

40 Card TR, Solaymani-Dodaran M, West J: Incidence and mortality of primary sclerosing cholangitis in the UK: a population-based cohort study. J Hepatol 2008;48:939-944.

41 Kingham JG, Kochar N, Gravenor MB: Incidence, clinical patterns, and outcomes of primary sclerosing cholangitis in South Wales, United Kingdom. Gastroenterology 2004; 126:1929-1930.

42 Lindkvist B, et al: Incidence and prevalence of primary sclerosing cholangitis in a defined adult population in Sweden. Hepatology 2010;52:571-577.

43 Kaplan G, et al: The burden of large and small duct primary sclerosing cholangitis in adults and children: a population-based analysis. Am J Gastroenterol 2007;102:1042-1049.

44 Boonstra K, et al: Population-based epidemiology, malignancy risk, and outcome of primary sclerosing cholangitis. Hepatology 2013;58:2045-2055.

45 Tanaka A: The epidemiological study of PSC in Japan. Health Labour Sciences Research Grant from Research on Measures for Intractable Diseases, the Intractable Hepato-Biliary Disease Study Group in Japan (in Japanese). 2008. 